\title{
Altered Cerebrospinal Fluid Concentrations of Hydrophobic and Hydrophilic Compounds in Early Stages of Multiple Sclerosis-Metabolic Profile Analyses
}

\author{
A. Podlecka-Piętowska ${ }^{1}$ - A. Kacka ${ }^{2,3}$ (D) B. Zakrzewska-Pniewska ${ }^{1} \cdot$ M. Nojszewska ${ }^{1} \cdot$ E. Zieminska $^{4}$. \\ M. Chalimoniuk ${ }^{5,6} \cdot$ B. Toczylowska ${ }^{7,8}$
}

Received: 8 February 2019 / Accepted: 7 May 2019 / Published online: 27 May 2019

(C) The Author(s) 2019

\begin{abstract}
The lack of a single predictive or diagnostic test in multiple sclerosis (MS) remains a major obstacle in the patient's care. The aim of this study was to investigate metabolic profiles, especially lipids in cerebrospinal fluid (CSF) using ${ }^{1} \mathrm{H}-\mathrm{NMR}$ spectroscopy and metabolomics analysis to discriminate MS patient group from the control ones. In this study, 19 MS patients and 19 controls, without neurological problems, patients were enrolled. To obtain the CSF metabolic profiles, NMR spectroscopy was used. Hydrophilic and hydrophobic compounds were analyzed using univariate and multivariate supervised analysis orthogonal partial least square discriminant analysis (OPLS-DA). Targeted OPLS-DA analysis of 32 hydrophilic and 17 hydrophobic compounds obtained 9 hydrophilic metabolites and 8 lipid functional groups which had the highest contribution to patient's group separation. Lower concentrations of CSF hydrophilic and hydrophobic compounds were observed in MS patients as compared to control group. Acetone, choline, urea, 1,3-dimethylurate, creatinine, isoleucine, myo-inositol, leucine, and 3-OH butyrate; saturated and monounsaturated acyl groups of $\omega-9, \omega-7, \omega-6, \omega-3$, and fatty acid, triglycerides, 1,3-DG, 1-MG, and unassigned component signal at $3.33 \mathrm{ppm}$ were the most important signal compounds in group separation. Analysis of metabolic profile of raw CSF and their lipid extract shows decreased levels of many compounds and led to the conclusion that MS patients could have a disturbance in many metabolic pathways perhaps leading to the decreased level of acetyl-CoA and/or inflammation. CSF metabolic profile analyses could be used as a fingerprint for early MS diagnosis.
\end{abstract}

Keywords Multiple sclerosis · Inflammation · Metabolomics · NMR spectroscopy

A. Kacka

agata.kacka2@gmail.com

A. Podlecka-Piętowska

a.podlecka@wum.edu.spl

E. Zieminska

elziem@imdik.pan.pl

M. Chalimoniuk

mchalimoniuk@imdik.pan.pl

B. Toczylowska

beata.toczylowska@ibib.waw.pl

1 Department of Neurology, Medical University of Warsaw, Zwirki i Wigury 61, 02-091 Warsaw, Poland

2 Department of Anesthesiology, Medical University of Warsaw, Zwirki i Wigury 61, 02-091 Warsaw, Poland
3 Department of Anesthesiology and Intensive Care, The Maria Skłodowska Curie Memorial Cancer Centre and Institute of Oncology, WK Roentgena 5, 02-781 Warsaw, Poland

4 Department of Neurochemistry, Mossakowski Medical Research Centre Polish Academy of Sciences, Pawinskiego Str. 5, 02-107 Warsaw, Poland

5 Department of Cellular Signaling, Mossakowski Medical Research Centre Polish Academy of Sciences, Pawinskiego Str. 5, 02-107 Warsaw, Poland

6 Department of Tourism and Health in Biala Podlaska, Józef Piłsudski University of Physical Education in Warsaw, Marymoncka 34, 00-968 Warsaw, Poland

7 Institute of Biocybernetics and Biomedical Engineering, Trojdena Str. 4, 02-109 Warsaw, Poland

8 NMR Laboratory, Institute of Biochemistry and Biophysics, Pawinskiego Str. 5A, 02-107 Warsaw, Poland 


\section{Introduction}

Multiple sclerosis (MS) is a chronic autoimmune disease that affects the central nervous system (CNS) characterized by demyelination and simultaneous axonal and neuronal degeneration that occurs from the earliest clinical stage of the disease (Campbell et al. 2011). Although the causes of MS are not completely known, it is notorious that this disease is characterized by heterogeneous and multifaceted mechanisms involving oxidative damage (Fischer et al. 2013), increased inflammation (Lubina-Dabrowska et al. 2017), and mitochondrial injury (Campbell and Mahad 2012). At the places of damaged axons and oligodendrocytes, the changes in the inflammation occur which cause the formation of active and inactive demyelinating plaques in the brain (Brosnan et al. 1996). In the demyelinating plaques, activated astrocytes, microglia, T cells, and macrophages occur which in turn secrete pro-inflammatory factors such as cytokines (IL-6, IL-1 $\beta$, $\mathrm{TNF} \alpha$, INF $\gamma$ ), free oxygen radicals (ROS), and nitric oxide (NO) (Brosnan et al. 1996; DeGroot et al. 1997). All the above-mentioned pro-inflammatory mediators are found to be elevated in the cerebral cortex, cerebrospinal fluid (CSF) as well as in the serum in MS patients. In $90 \%$ of patients with MS, local disturbances of B cells' response elicit presence of oligoclonal bands in CSF which have been proposed as a helpful biomarker for MS diagnosis and evaluation of treatment. Their presence discloses the intrathecal immunoglobulin G (IgG) synthesis (Bo et al. 1994). However, recent studies have indicated a large number of controversies about the oligoclonal IgG bands' role in MS (O'Connor et al. 2003). Currently, diagnosis of MS is based on clinical criteria including symptoms, magnetic resonance imaging (MRI), lumbar puncture to identify inflammatory proteins, and excluding other disorders (Zhou et al. 2016; Hunter 2016; Raphael et al. 2015). For many patients, diagnosis takes months, and the decision of introduction of MS therapy is delayed. The lack of a single predictive or diagnostic test in MS remains a major obstacle in the patient's care. In recent years, there have been advances in molecular biology, cellular immunology, and "omics" (genomics, proteomics, metabolomics) which focus on exploring the processes underlying disease pathogenesis to provide a list of possible MS biomarkers (Kuhle et al. 2015; Poddighe et al. 2017).

In the last decade, advances in high-throughput approaches allowed development of proteomic and metabolomics studies in evaluating the association of genetic and phenotypic variability with disease sensitivity and therapy response. These considerations have more value in the case of MS, a multifactorial disease with high heterogeneity in clinical course, and treatment response.

Metabolomics concerns the identification and quantification of small endogenous molecules in a biological system. Because the metabolite represents substrates and the final products of physiological processes in a living organism, the profiling of the metabolome in tissues and biofluids offers an instantaneous molecular image of the phenotype. Among the several analytical techniques, HPLC, highresolution mass spectrometry, and gas chromatography coupled with mass spectrometry are the most commonly used methods in the metabolomics filed (Kim et al. 2017; Del Boccio et al. 2016; Poisson et al. 2015; Dickens et al. 2015; Cha et al. 2015; Pieragostino et al. 2018). These techniques have been used to investigate MS in serum and CSF hydrophilic and hydrophobic compounds (Bhargava and Calabresi 2016; Cocco et al. 2016; Moussallieh et al. 2014; Reinke et al. 2014). Nuclear magnetic resonance (NMR) spectroscopy is not often presented in CSF metabolomics of MS patients. In CSF studies of most research groups, other neurological disease (OND) patients have been used as the control group. The participation of metabolomics in the autoimmune process of MS has been examined in serum, but the role of lipids, especially in combination with amino acids, remains poorly defined. Lipids play a main dual role in MS, both as substrates of myelin and as mediators of inflammation.

The aim of this study was to investigate metabolic profiles, especially lipids in CSF using ${ }^{1} \mathrm{H}-\mathrm{NMR}$ spectroscopy and metabolomics analysis to discriminate the MS patient group from the control ones. Our studies focused on metabolic and lipid profiles of the same CSF sample and compared the results of MS patients to control, non-neurological problem patients. We hypothesized that disturbances in CSF metabolite profiles reflected the myelin degradation/regeneration during the inflammatory process of brain tissue in MS relapsing/ remitting status.

\section{Materials and Methods}

\section{Patients}

In this study, 19 patients (13 females and 6 males) with MS according to the McDonald criteria of 2010 (Polman et al. 2011) were enrolled in the study in 2016-2017. Patients were admitted to the department of neurology for MS diagnosis. All patients underwent extensive neurologic evaluation; impairment and disability were measured using the Expanded Disability Status Scale (EDSS). None of them had a history of immunomodulatory or immunosuppressive therapy. All patients had MRI to show characteristic multiple lesions in the periventricular and subcortical white matter of the brain as well as gadolinium enhancements, a result of active demyelination. We excluded patients with any other chronic diseases: depression, diseases of liver, kidney, thyroid gland, and abnormalities in blood morphology. The analysis of CSF obtained from all the patients was done after setting the 
first diagnosis of MS. Based on the clinical data, all MS patients had an active disease status.

The control group of 19 patients (12 females and 7 males) was set from those who underwent minor vascular surgery or inguinal hernia repair under spinal anesthesia during the last 2 years and had not any neurological problems.

At the time of screening for trial inclusion, potential participants received thorough written and oral information on the purpose and duration of the study, as well as possible adverse events, and signed the informed consent. The study was designed in accordance with the Declaration of Helsinki. The study protocol was approved by the Hospital Bioethics Committee.

\section{Sample Preparation and Spectra Acquisition}

Two milliliters of CSF were collected from lumbar puncture during anesthesia or clinical examination. All samples were centrifuged at room temperature at 15,000 rpm for $5 \mathrm{~min}$, and the supernatants were frozen at $-80{ }^{\circ} \mathrm{C}$ until NMR analysis was performed. The $\mathrm{pH}$ was stabilized at $7.4 \pm 0.2$ using $\mathrm{HCl}$. To achieve a stable lock signal, a $100 \mu \mathrm{l}$ of $\mathrm{D}_{2} \mathrm{O}$ was added to each sample volume. 3-Trimethylsilyl propionic acid (TSP) with a final concentration in the sample of $1 \mathrm{mM}$ was used as an internal reference compound for the normalization of all spectra, quantitative statistical analysis. Hydrophobic compounds were prepared according to the procedure described in our past publication (Zieminska et al. 2018).

All NMR spectra were acquired at $25^{\circ} \mathrm{C}$ on a Varian Inova 400 (Varian Inc.) spectrometer. One pulse sequence was applied to hydrophilic and hydrophobic CSF compounds in ${ }^{1} \mathrm{H}-$ NMR examinations. Settings for each measurement were for raw and $\mathrm{CDCl}_{3}$ samples: 512/128 transients, $12 / 5 \mathrm{~s}$ pulse repetition time, respectively. Zero-filling to $16 \mathrm{k}$ data points, line broadening of 0.5 , baseline and phase correction were applied to each spectrum using software implemented in the spectrometer. Signals were assigned according to our own reference database and literature information (Lutz et al. 1998; Wevers et al. 1995).

\section{Data Analysis}

Quantities of metabolites were expressed as relative intensity. Spectra were both baseline and phase corrected and normalized to the TSP or $\mathrm{CDCl}_{3}$ rest signal prior to statistical analysis. For the statistical analysis of raw CSF samples and lipid spectra, 32 and 18 signals of the NMR spectrum were selected, respectively.

Univariate statistical analysis was performed for all data using the Mann-Whitney test followed by Tukey correction. A $P$ value lower than 0.05 was considered as significant.

Multivariate statistical analysis was performed using supervised methods of orthogonal partial least square discriminant analysis (OPLS-DA). In the OPLS-DA modeling, the goodness of fit is reported as the cumulative score across all of the components R2cum - explained by the model and Q2cumpredicted by the model. OPLS-DA model was considered significant if R2cum and Q2cum were significantly larger than zero and was considered as good when both values were equal or greater than 0.5 (Bylesjo et al. 2008). The variable importance in the projection (VIP) value of each variable in the model was calculated to indicate its contribution to the classification. Those variables with VIP value greater than 1.0 were considered significantly different, and a larger VIP value of a variable represented a higher contribution to the discrimination between groups (Karp et al. 2005; Toczylowska et al. 2013). A receiver-operator characteristic (ROC) curve was created by plotting the true positive rate (TPR) versus the false positive rate $(\mathrm{FPR}=1-\mathrm{TNR})$ at various threshold settings of the criterion parameter. As a quantitative measure of the classification success, the area under the ROC-AUC was calculated. Multivariate analysis, OPLS-DA, was performed using the software package SIMCA-P (Version 14, MKS Umetrics, Sweden) (Ellis et al. 2007).

\section{Results}

The MS group of patients had an average age of 34.2 \pm 9.6 , with median 2.5 years of disease (1-15 years), median EDSS value was $1.75(0-3.5)$, the median number of relapses before admission was $1(0-3)$ (Table 1$)$. All the patients had been diagnosed with at least one active lesion in the brain (9 out of 19) and/or in the spinal cord (12 out of 19). Routine CSF examinations showed cytosis as well as oligoclonal $\mathrm{IgG}$ bands in all patients.

\section{The Concentrations of CSF Hydrophilic Compounds}

All 32 hydrophilic compounds selected for the identification of potential differences in biochemical composition caused by neuronal death processes, the obtained $P$ value for univariate analysis, and VIP value $>1$ are presented in Table 2 .

The multivariate OPLS-DA modeling was employed using the knowledge of patient's classification. The best OPLS-DA model consisted of one predictive and seven orthogonal components $(\mathrm{R} 2 \mathrm{cum}=0.948, \mathrm{Q} 2 \mathrm{cum}=0.703)$. In this model, $100 \%$ of all patients were classified correctly according to their groups (Fig. 1). The most important parameters (VIP > 1) that contributed to class separation were the NMR signals from acetone, choline, urea, 1,3-dimethylurate, creatinine, isoleucine, myo-inositol, leucine, and 3-OH-butyrate. The model was tested for validity by applying the analysis of variance to cross-validated predictive residuals ( $F$ test, $P=0.004$ ). In the MS group, all metabolites had lower concentrations as compared to the control group (Figs. 2 and 3). A receiver-operator 
Table 1 Clinical parameters of control and MS groups

\begin{tabular}{lll}
\hline Parameter & Control & MS \\
\hline N (F/M) & $19(12 / 7)$ & $19(13 / 6)$ \\
Age & $46.2 \pm 12.7$ & $34.2 \pm 9.6$ \\
EDSS value (median/min/max) & - & $1.75(0-3.5)$ \\
Number of relapses (median (min-max)) & - & $1(0-3)$ \\
Cytosis (number $\left./ \mathrm{mm}^{3}\right)$ & $1.63 \pm 0.33$ & $3.74 \pm 2.83 *(p=0.009)$ \\
Protein $(\mathrm{mg} \%)$ & $26.44 \pm 3.97$ & $39.12 \pm 10.82 *(p<0.001)$ \\
Glucose $(\mathrm{mg} / \mathrm{dL})$ & $57.89 \pm 7.5$ & $57.50 \pm 11.46$ \\
\hline
\end{tabular}

*Significant differences between groups
Table 2 Direction of changes and statistical significance of analyzed hydrophilic compounds of CSF fluid for MS vs control group

\begin{tabular}{|c|c|c|c|c|c|}
\hline \multicolumn{2}{|c|}{ Compound } & \multirow[t]{2}{*}{ MS vs control } & \multirow[t]{2}{*}{$P$ value } & \multirow[t]{2}{*}{ VIP value } & \multirow[t]{2}{*}{ Published results } \\
\hline ppm & Assignment & & & & \\
\hline 8.46 & Formate & $\downarrow$ & 0.550 & & \\
\hline 7.73 & Hist & $\downarrow$ & 0.261 & & \\
\hline 7.39 & Phe & $\downarrow$ & 0.389 & & \\
\hline 6.86 & Tyr & $\uparrow$ & 0.872 & & \\
\hline 5.76 & Urea & $\downarrow$ & 0.006 & 1.57 & $\begin{array}{l}\downarrow(\text { Sinclair et al. 2010; } \\
\quad \text { Koneczny et al. 2014) }\end{array}$ \\
\hline 4.12 & Lactate & $\downarrow$ & 0.457 & & \\
\hline 4.07 & Myo-inositol & $\downarrow$ & 0.015 & 1.13 & $\downarrow$ (Sinclair et al. 2010) \\
\hline 4.01 & Betaine & $\downarrow$ & 0.804 & & \\
\hline 3.52 & Gly & $\downarrow$ & 0.782 & & \\
\hline 3.36 & 1,3 dimethylurate & $\downarrow$ & 0.358 & 1.45 & $\downarrow$ (Sinclair et al. 2010) \\
\hline 3.31 & Cysteine & $\downarrow$ & 0.274 & & \\
\hline 3.25 & Glucose & $\downarrow$ & 0.693 & & \\
\hline 3.21 & Choline & $\downarrow$ & 0.008 & 1.82 & $\downarrow$ (Sinclair et al. 2010) \\
\hline 3.16 & Citrulline & $\downarrow$ & 0.166 & & \\
\hline 3.05 & Creatinine & $\downarrow$ & 0.007 & 1.19 & $\downarrow$ (Sinclair et al. 2010) \\
\hline 3.04 & Creatine & $\downarrow$ & 0.140 & & \\
\hline 2.68 & Citrate & $\downarrow$ & 0.569 & & \\
\hline 2.42 & Gln & $\downarrow$ & 0.872 & & \\
\hline 2.38 & Pyruvate & $\downarrow$ & 0.550 & & \\
\hline 2.28 & Acetoacetate & $\downarrow$ & 0.530 & & \\
\hline 2.11 & Methionine & $\downarrow$ & 0.550 & & \\
\hline 2.24 & Acetone & $\downarrow$ & 0.001 & 2.10 & $\downarrow$ (Sinclair et al. 2010) \\
\hline 2.13 & Gln & $\downarrow$ & 0.362 & & \\
\hline 2.03 & $\mathrm{~N}$-acetyl $\mathrm{NH}_{3}$ group & $\downarrow$ & 0.942 & & \\
\hline 1.93 & Acetate & $\downarrow$ & 0.942 & & \\
\hline 1.72 & Lys & $\downarrow$ & 0.872 & & \\
\hline 1.47 & Ala & $\downarrow$ & 0.849 & & \\
\hline 1.34 & Lactate & $\downarrow$ & 0.511 & & \\
\hline 1.21 & 3-OH-butyrate & $\downarrow$ & 0.157 & 1.12 & $\downarrow$ (Sinclair et al. 2010) \\
\hline 1.03 & Val & $\downarrow$ & 0.257 & & \\
\hline 0.96 & Ile & $\downarrow$ & 0.140 & 1.09 & $\downarrow$ (Sinclair et al. 2010) \\
\hline 0.9 & Leu & $\downarrow$ & 0.052 & 1.18 & $\downarrow$ (Sinclair et al. 2010) \\
\hline
\end{tabular}


Fig. 1 The score plot of the twocomponent OPLS-DA model for hydrophilic compounds of NMR data; to[1] represents within class variation in the first orthogonal component, whereas $\mathrm{t}[1]$ represents between class variation in the first predictive component. Ellipse represents Hotelling T2 with $95 \%$ confidence in score plots

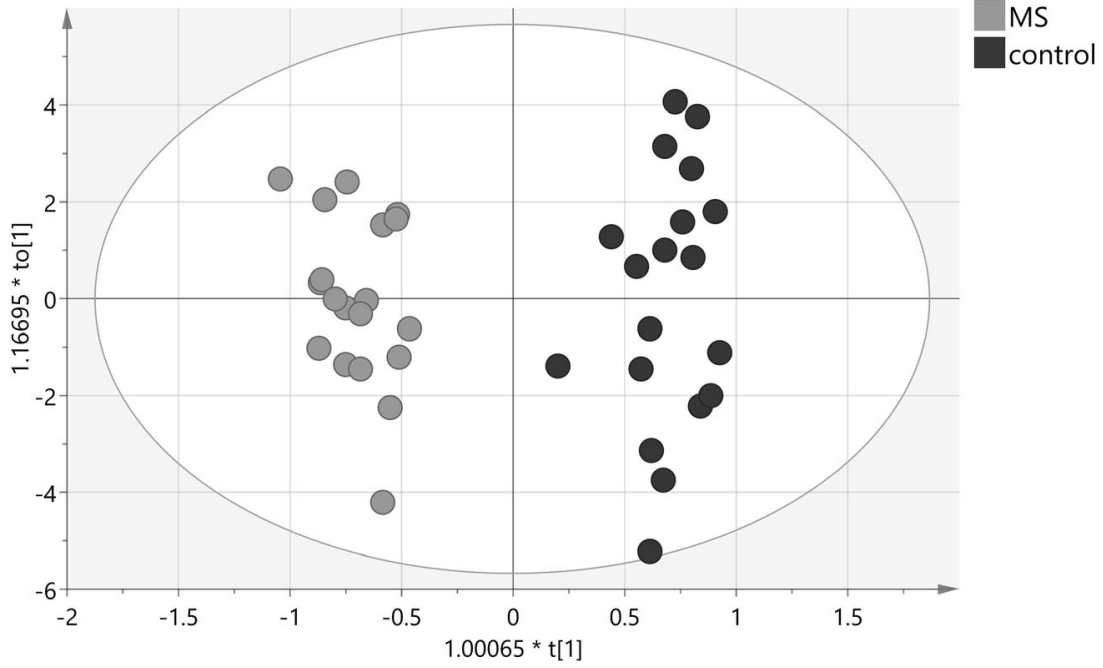

characteristic (ROC) generated from the ratio of the sensitivity to 1 -selectivity resulted in an area under the curve of 1.0 for both, control and MS groups, which was the perfect classification (Fig. 4).

\section{The Concentration of CSF Hydrophobic Compounds}

Seventeen lipid compound functional groups from proton NMR signals are presented in Table 3 with $P$ value of univariate analysis and VIP $>1$ values. Discriminant analysis, OPLS-DA, of the lipid compound data allowed building a model (Fig. 5). The model consisted of one predictive and four orthogonal components $(\mathrm{R} 2 \mathrm{cum}=0.787, \mathrm{Q} 2 \mathrm{cum}=$ $0.528)$. In this model, $92 \%$ of all patients were classified correctly according to their groups (12 out of 19 in the MS group and 18 out of 19 in the control group). The most important parameters (VIP $>1$ ) that contributed to class separation were the signals from $-\mathrm{CH}_{3}-$ saturated, monounsaturated $\omega-9$ and/ or $\omega-7$ acyl groups and fatty acid (FA) $(0.86 \mathrm{ppm}),-\mathrm{CH}_{3}-$ unsaturated $\omega-6$ acyl groups and FA $(0.88 \mathrm{ppm}),-\mathrm{CH}_{3}$ unsaturated $\omega-3$ acyl groups and FA $(0.96 \mathrm{ppm}),-\mathrm{CH}_{2}-$ $\mathrm{CH}=\mathrm{CH}-$ acyl groups and $\mathrm{FA}(1.99 \mathrm{ppm}),-\mathrm{OCO}-\mathrm{CH}_{2}-\mathrm{acyl}$ groups in triglyceride (TG) $(2.25 \mathrm{ppm}),-\mathrm{OCO}-\mathrm{H}_{2-},-\mathrm{COOH}-$ $\mathrm{CH}_{2}-$ acyl groups in 1,3-DG, 1-MG, and FA (2.35 ppm), $\mathrm{ROCH}_{2}-\mathrm{CHOH}-\mathrm{CH}_{2} \mathrm{OH}$ glyceryl group in $1-\mathrm{MG}$ (3.68 ppm) and unassigned signal at $3.33 \mathrm{ppm}$. The model was tested for validity by applying the analysis of variance to cross-validated predictive residuals ( $F$ test, $P=0.02$ ). In the MS group, all compounds had a lower concentration as compared to control group except unassigned functional group signal (singlet) at $3.68 \mathrm{ppm}$ and $=\mathrm{HC}-\mathrm{C}_{2}-\mathrm{CH}=$ diunsaturated $\omega-6$ acyl groups and FA that had a higher concentration as compared to control group (Figs. 6 and 7). A receiver-operator characteristic (ROC) generated from the ratio of the sensitivity to 1 -selectivity resulted in an area under the curve of 0.94 for both, control and MS groups, which was the almost perfect classification (Fig. 8).

NMR signal: $s$ singlet, $m$ multiplet
Fig. 2 Score contribution for hydrophilic compounds for MS group versus control group. Black color indicated metabolites with VIP $>1$

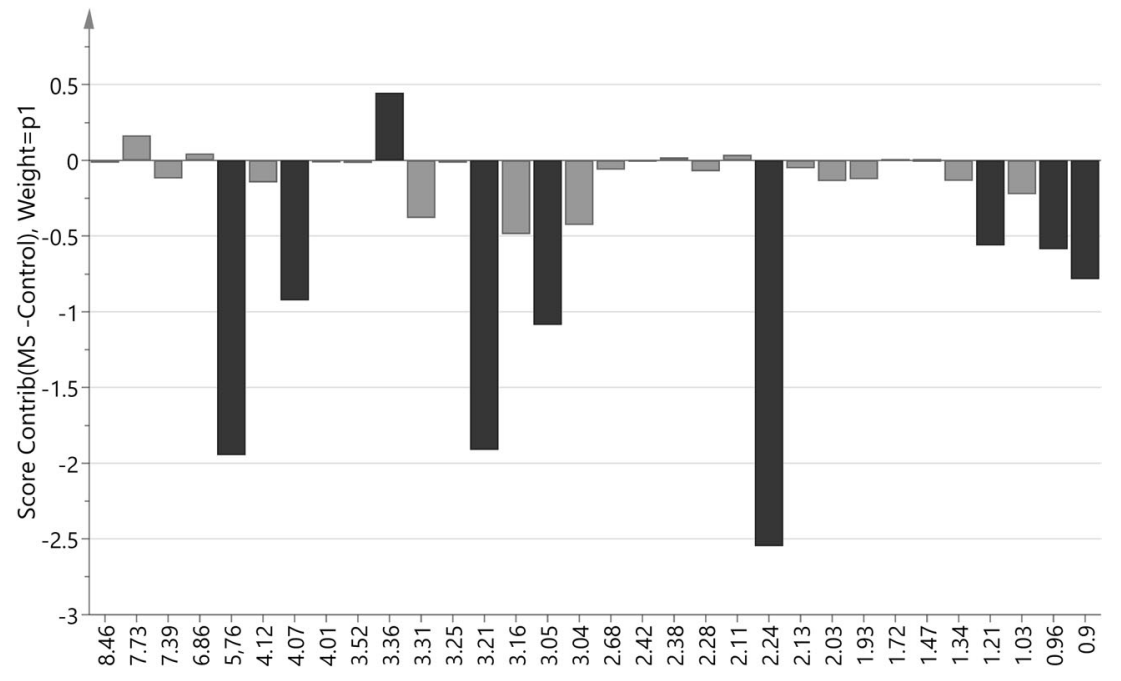



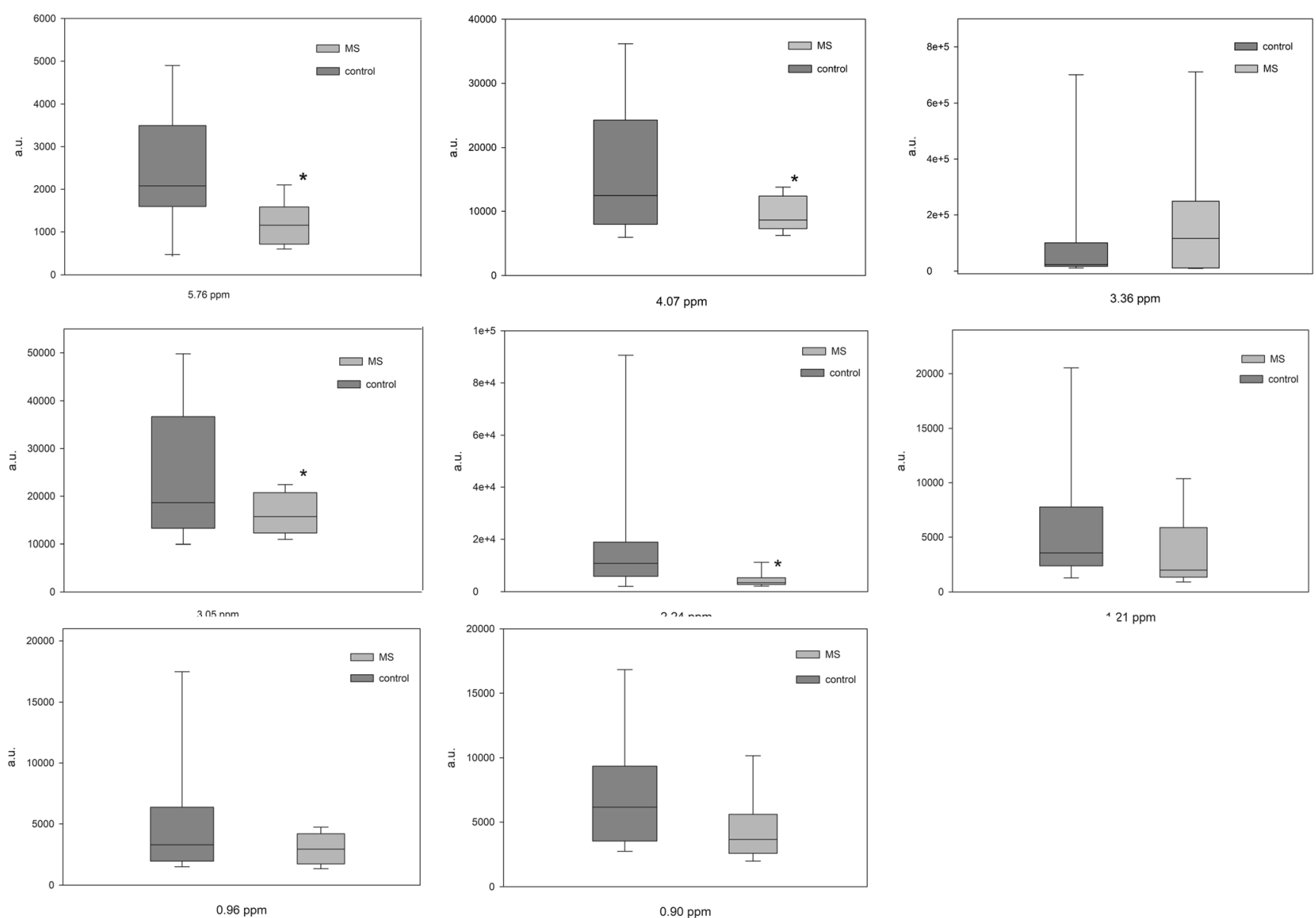

Fig. 3 Box and Whisker plot of hydrophilic compounds of VIP $>1$ compounds. “*” indicated differences with $p<0.05$ (Mann-Whitney test)

\section{Discussion}

In this study, investigators performed metabolic and lipids profiling using ${ }^{1} \mathrm{H}-\mathrm{NMR}$ spectroscopy of CSF samples from the early stage of MS to define the differences between MS and control-matched subjects. This approach can detect a number of endogenous metabolites that influence the condition to change an organism in MS. Thus, identification of the important metabolites allows evaluation of potential pathways characterizing an early stage of the disease. The model created in this study revealed differences in the metabolites and lipids of CSF in the two groups. Therefore, the authors looked at a
Fig. 4 The ROC curve of the true positive rate (TPR) versus the false positive rate $(\mathrm{FPR}=1-\mathrm{TNR})$ and ROC-AUC values for both groups as quantitative measure of the classification success for hydrophilic compounds for the OPLS-DA model; sensitivity: $100 \%, 95 \% \mathrm{CI}=82.35 \%$ to $100.00 \%$; specificity: $100 \%, 95 \%$ $\mathrm{CI}=82.35 \%$ to $100.00 \%$

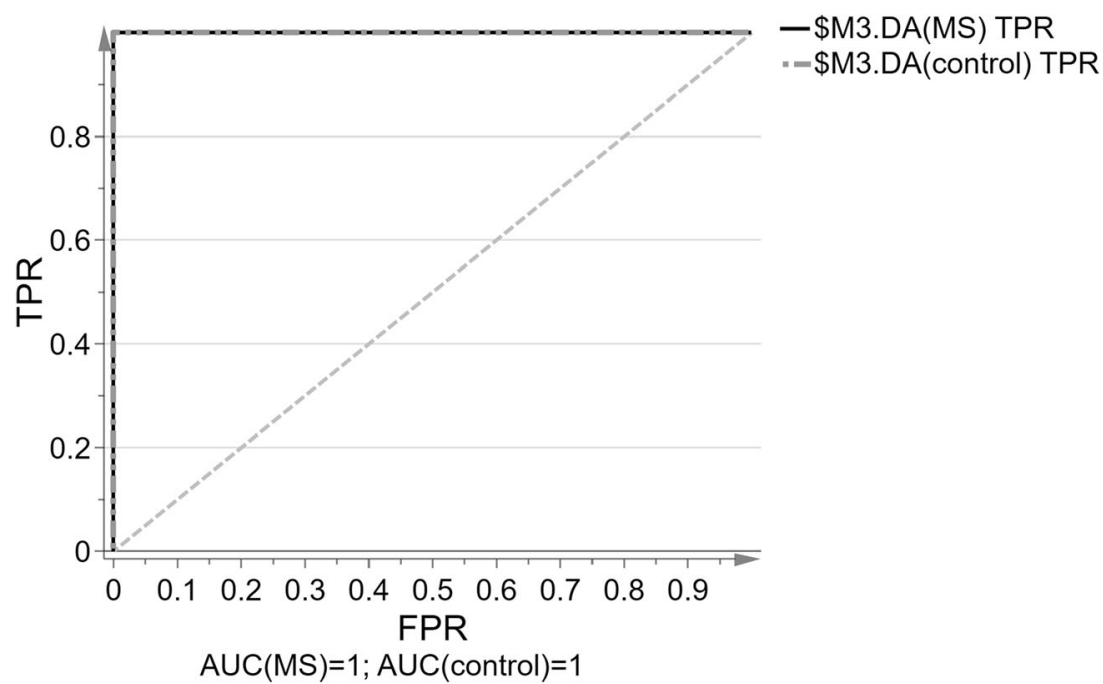


Table 3 Direction of changes and statistical significance of analyzed hydrophobic functional groups of CSF fluid for MS vs control group

Compound $\quad$ MS vs control $P$ value VIP value Publish results

\begin{tabular}{|c|c|c|c|c|c|}
\hline ppm & Functional group & & & & \\
\hline 6.10 & Estriol $(-\mathrm{HC}(2,4)=)$ & $\downarrow$ & 0.378 & & \\
\hline 5.34 & $-\mathrm{HC}=\mathrm{CH}-$ in $\mathrm{FA}$ & $\downarrow$ & 0.608 & & \\
\hline 4.70 & Unassigned 1 & $\downarrow$ & 0.222 & & \\
\hline $4.17-4.08$ & $\mathrm{ROCH}_{2}-\mathrm{CHOH}-\mathrm{CH}_{2} \mathrm{OR}^{\prime}$ in glyceryl group of $1,3-\mathrm{DG}$ & $\downarrow$ & 0.457 & & \\
\hline $3.68_{\mathrm{m}}$ & $\mathrm{ROCH}_{2}-\mathrm{CHOH}-\mathrm{CH}_{2} \mathrm{O}$ in glyceryl group of $1-\mathrm{MG}$ & $\downarrow$ & 0.107 & 1.09 & $\downarrow$ (Pietrocola et al. 2015) \\
\hline $3.68_{\mathrm{s}}$ & Unassigned 2 & $\uparrow$ & 0.734 & & \\
\hline 3.50 & Pregnenolon & $\downarrow$ & 0.522 & & \\
\hline 3.33 & Unassigned 3 & $\downarrow$ & 0.854 & 1.04 & \\
\hline 2.81 & $=\mathrm{HC}-\mathrm{CH}_{2}-\mathrm{CH}=$ in acyl groups of diunsaturated $\omega-6$ and FA & $\uparrow$ & 0.861 & & \\
\hline 2.35 & $-\mathrm{OCO}-\mathrm{CH}_{2-},-\mathrm{COOH}-\mathrm{CH}_{2}-$ in acyl groups of $1,3-\mathrm{DG}, 1-\mathrm{MG}$ and $\mathrm{FA}$ & $\downarrow$ & 0.082 & 1.04 & $\downarrow$ (Pietrocola et al. 2015) \\
\hline 2.25 & $-\mathrm{OCO}-\mathrm{CH}_{2}-$ in acyl groups of FA & $\downarrow$ & 0.243 & 1.34 & $\downarrow$ (Pietrocola et al. 2015) \\
\hline 1.99 & $-\mathrm{CH}_{2}-\mathrm{CH}=\mathrm{CH}-$ in acyl groups of $\mathrm{FA}$ & $\downarrow$ & 0.492 & 1.08 & $\downarrow$ (Pietrocola et al. 2015) \\
\hline 1.63 & $-\mathrm{OCO}-\mathrm{CH}_{2}-\mathrm{CH}_{2}-$ in acyl groups of 1,3-DG, $1-\mathrm{MG}$ and FA & $\downarrow$ & 0.249 & & \\
\hline 1.24 & $-\left(\mathrm{CH}_{2}\right)_{\mathrm{n}}-$ in acyl groups of FA & $\downarrow$ & 0.013 & & \\
\hline 0.96 & $-\mathrm{CH}_{3}$ in acyl groups of unsaturated $\omega-3 / \mathrm{FA}$ & $\downarrow$ & 0.060 & 1.12 & $\downarrow$ (Pietrocola et al. 2015) \\
\hline 0.88 & $-\mathrm{CH}$ in acyl groups of unsaturated $\omega-6 / \mathrm{FA}$ & $\downarrow$ & 0.001 & 1.28 & $\downarrow$ (Pietrocola et al. 2015) \\
\hline 0.86 & $-\mathrm{CH}_{3}$ in acyl groups of saturated, monounsaturated $\omega-9$ and/or $\omega-7$ and FA & $\downarrow$ & 0.003 & 1.34 & $\downarrow$ (Pietrocola et al. 2015) \\
\hline
\end{tabular}

possible correlation in the metabolic profiles of hydrophilic and hydrophobic compounds with clinical parameters to study their relationship with pathology. The most important problem in comparing the results of different research groups is the use of different control groups. Most groups have used OND patients as the control group. In our study, we used nonneurological problem patients as the control group.

By targeted analysis of OPLS-DA, 32 hydrophilic metabolites and 17 hydrophobic compound functional groups were studied. Investigators obtained nine NMR signals associated with hydrophilic metabolites and eight NMR signals associated with lipid functional groups which had the highest contribution to patient's group separation.

Lower concentrations of CSF hydrophilic compounds were observed in MS patients as compared to control group. Univariate analysis indicated significant differences in urea, myo-inositol, 1,3-dimethylurate, choline, creatinine, and acetone concentrations while OPLS-DA indicated the low significant concentration of acetone, choline, urea, 1,3dimethylurate, creatinine, isoleucine, myo-inositol, leucine, and 3-OH butyrate as in other group studies (Sinclair et al. 2010). Noga et al. (2012) have demonstrated a significant
Fig. 5 The score plot of the twocomponent OPLS-DA model for hydrophobic compounds for NMR data; to[1] represents within class variation in the first orthogonal component, whereas $\mathrm{t}[1]$ represents between class variation in the first predictive component. Ellipse represents Hotelling T2 with 95\% confidence in score plots

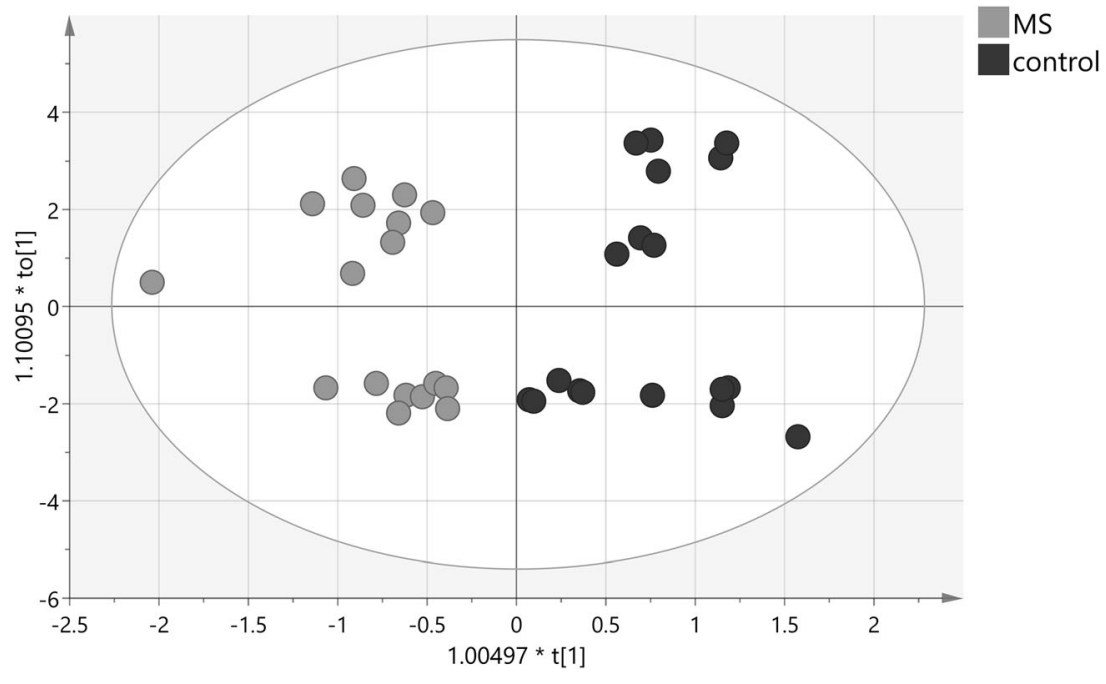


Fig. 6 Score contribution for hydrophobic compounds for MS group versus control group. Black color indicated metabolites with $\mathrm{VIP}>1$

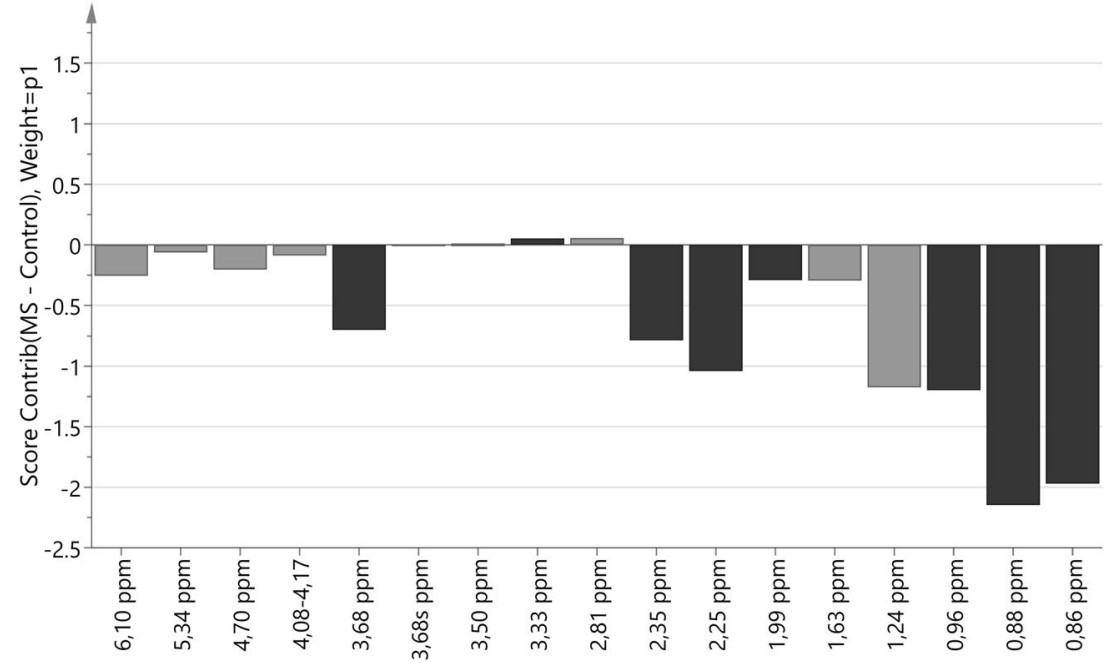

change in amino acid metabolism in CSF during EAE. They found the decreased level of metabolites related to pathways including NO synthesis, energy metabolism, polyamine synthesis, and levels of endogenous antioxidants. Sinclair et al. (2010) have shown a similar change in the metabolite levels in CSF as was observed in the present study. The metabolic changes of MS may be related to altered energy metabolism and FA biosynthesis in the brain. Downregulation of citrated and acetate may support disruption of TCA cycle through pyruvate pathway. This was confirmed by the reduced metabolism of lipid compounds in CSF in this study.

Acetone is an end product of ketosis, a metabolic state that produces ketone bodies for use as another fuel for the brain.
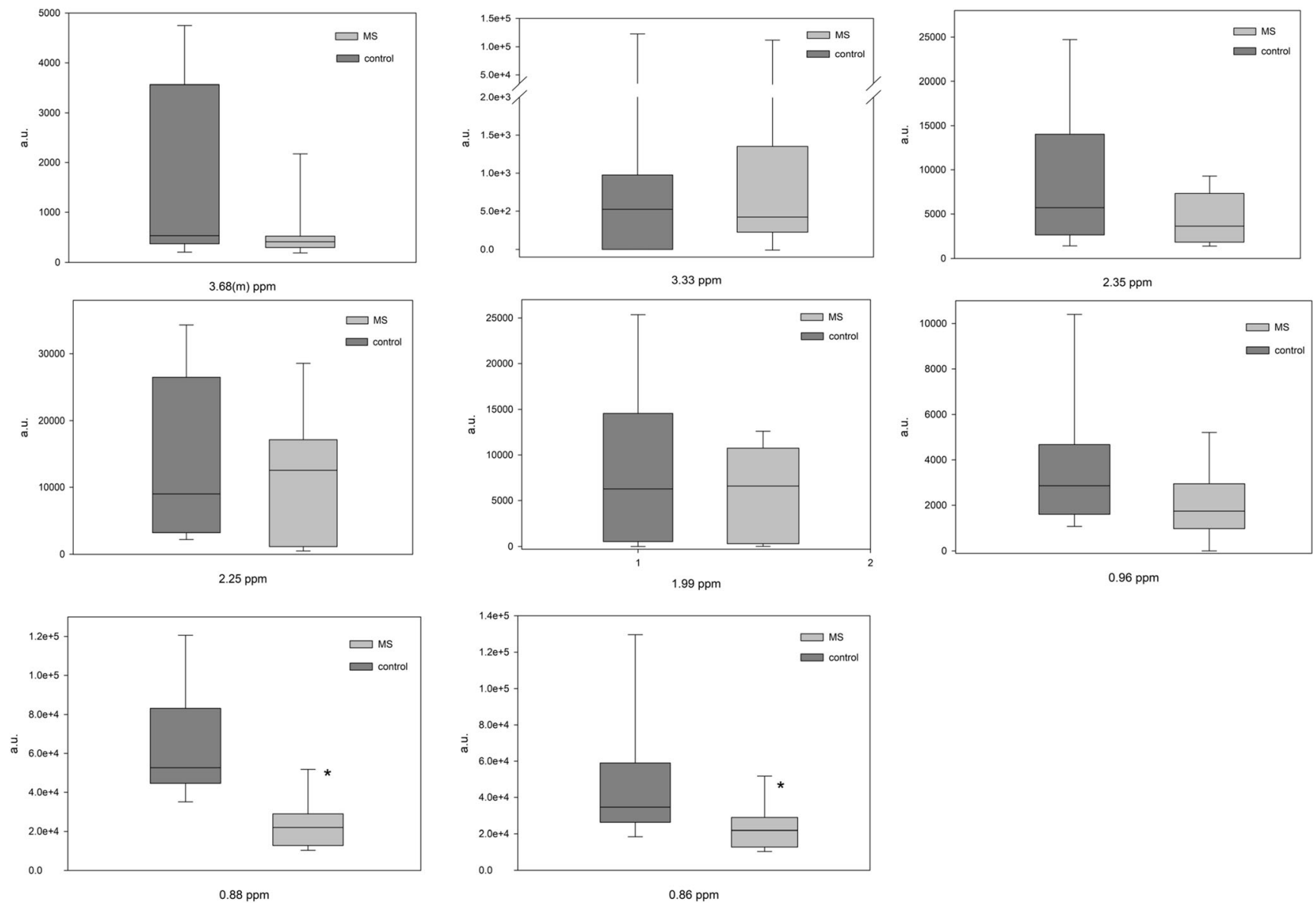

Fig. 7 Box and Whisker plot for hydrophobic compounds of VIP $>1$ NMR signals. “*” indicated differences with $p<0.05$ (Mann-Whitney test) 
Fig. 8 The ROC curve of the true positive rate (TPR) versus the false positive rate $(\mathrm{FPR}=1-\mathrm{TNR})$ and ROC-AUC values for both groups as quantitative measure of the classification success for hydrophobic compounds for the OPLS-DA model; sensitivity: $92.3 \%, 95 \% \mathrm{CI}=63.97 \%$ to $99.81 \%$; specificity: $72 \%, 95 \%$ $\mathrm{CI}=50.61 \%$ to $87.93 \%$

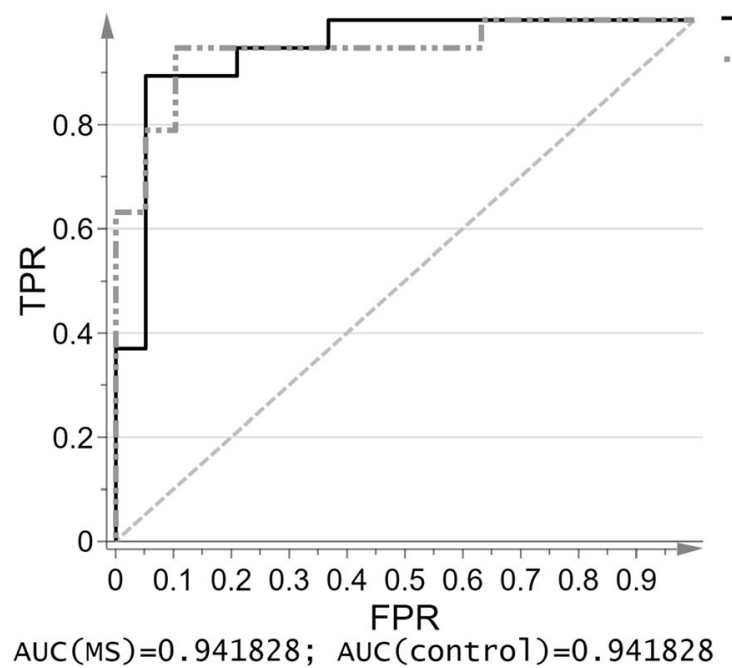

-\$M9.DA(MS) TPR - $\$$ M9.DA(control) TPR
Both the levels of 3-hydroxybutyrate (non-significant $P<0.157)$ and acetone were altered in patients as compared to the control subjects in this analysis. The significant decrease of acetone in CSF might imply that the decreased flux from acetyl-CoA into acetoacetyl-CoA resulted in lower production of acetone or a reduced efflux through the blood-brain barrier (BBB) and higher consumption in the brain. Interestingly, the expression level of GULT 1, a major glucose transporter in the $\mathrm{BBB}$, is down-regulated in the brain lesion of MS patients. Consistently, the observations for disturbed energy generation in CNS diseases including MS have been reported: mitochondrial dysfunctions detected in MS lesions as well as OND (Joseph et al. 2009; Ronowska et al. 2018).

On the analysis of lipid levels of CSF, especially saturated and unsaturated FAs, we observed their decrease in MS patient group as compared to the control object group. FAs are structural compounds that are components for cell membrane building. They are synthesized from cytosol compounds and acetyl-CoA from cytosol or mitochondria. In NMR experiments, used in these studies, it is not possible to measure very low concentrations of acetyl-CoA because it is below the sensitivity level of this method. However, we can measure the levels of compounds which are the products of cycles with acetyl-CoA participation which is necessary to synthesize FA and ketone bodies. We observed the decrease of FA and PUFA levels which could be the effect of altered level of acetyl-CoA (Pietrocola et al. 2015). In this study, we observed the decreased levels of ketone bodies (acetone, 3-OH butyric acid) that are indirect metabolites of decarboxylation of acetoacetate in the lipid degradation process. It could be the result of glycolysis cycle where acetyl-CoA participates. Low level of ketone bodies inhibits dopamine secretion (Cornille et al. 2010).

In our study, we observed altered choline and urea levels in the MS group as compared to the control group. Choline in the brain is an essential component in cholesterol and other lipids metabolism. The decrease of choline level influences their metabolism, further reducing their levels, similar to what we observed in our study. It also influences the so-called integrity and fluidity of the cell membrane (Zhong et al. 2014). Choline is a substrate for the synthesis of acetylcholine (ACh), excitation neurotransmitter. All immune cells have receptors for many neurotransmitters including ACh and neuropeptides. Immune cells stimulate the immune and brain system, and thus, disturbances in ACh influence their function. This may result in immunological disturbances including possible autoimmune reaction. The level of urea, the end compound of protein degradation cycle, is decreased in neuromuscular diseases, e.g., myasthenia gravis and dystrophies (Koneczny et al. 2014).

Another low-level compound in MS group is myo-inositol. It is partially synthesized in the brain and is an important part of glycolipids and cell membrane building compounds. It sensitizes serotonin and GABA receptors (Balla 2013).

We also observed decreased CSF creatine level in the MS patient group. Creatine takes part in phosphocreatine metabolism which in turn is the main energetic substrate for cells. Their decrease indicates energetic disturbances in neuronal cells. Brewer (Brewer and Wallimann 2000) demonstrated the neuroprotective role of creatine in their studies on rat hippocampus. The decrease of creatine level and thus energetic deficiency can correlate with MS neuronal deficiencies. This hypothesis was supported by the observed decreased levels of two ketogenic amino acids: leucine and isoleucine. Metabolism of ketogenic amino acids leads to the formation of acetyl-CoA or acetoacetyl-CoA. If they will not be completely utilized in the TCA cycle, the rest may undergo ketogenesis.

The most relevant results of our studies were the detection of an altered level of specific hydrophobic functional groups in the MS group compared to the control subjects. In particular, we found a significant decrease level in the MS group of 
saturated, monounsaturated acyl groups of $\omega-9$ and/or $\omega-7$, $\omega-6, \omega-3$, and FA, TG, 1,3-DG, 1-MG, glycerol group in 1$\mathrm{MG}$, and unassigned component signal at $3.33 \mathrm{ppm}$.

Pieragostino (Pieragostino et al. 2015) has analyzed the hydrophobic metabolites of MS and OND patients' CSF using MALDI-TOF mass spectrometry. This method needs special preparation of the samples (internal labeled standards) and therefore, is more complicated and more expensive. Our results differed from those obtained by the Pieragostino study because different control groups were examined (healthy vs. OND). The most relevant result from their studies is the altered levels of specific phospholipids in the MS group compared to the OND group. In particular, they reported a significantly increased level of lysophosphocholine (LPC) (18:1), (18:0), lysophosphoinositol (16:0) in the MS patients. LPCs are well correlated to Link index (also known as IgG Index) which is the parameter indicating high levels of intrathecal IgG synthesis. Intrathecal IgG synthesis is a common event in part of MS. In our patients, IgG index was elevated in CSF. We also obtained the down expression of lipid compounds in the CSF of MS group, a cerebral component suggestive for a possible function of these lipids as candidate biomarkers, reflecting intrathecal synthesis IgG and CNS inflammation.

Clinical and MRI studies indicate that axonal damage predominantly appears in the early MS and develops as a consequence of inflammatory process (Bendfeldt et al. 2009), leading to the most numerous ( $~ 85 \%$ of cases) relapsing-remitting form of the disease (Weiner 2008). Elevated levels of aforementioned pro-inflammatory cytokines and lipid peroxidation in the plasma, cerebrospinal cord, and brain cortex have been found in the patients with MS (Gonsette 2008; Keller and Mattson 1998); a positive correlation has been found between their levels and disease's activity and severity (Sharief 1991; Navikas and Link 1996). Gonzalo et al. (Gonzalo et al. 2012) performed targeted lipidome analyses comprising several oxidized phospholipids, lipid peroxidation-derived aldehydes, oxysterols, and oxidized lipids. The results confirmed the presence of aldehyde in agreement with data by NegreSalvayre (Negre-Salvayre et al. 2010) in human MS showing increased lipid peroxidation in serum. Lipid peroxidation can exert part of its pathological properties through modification of the protein. Lipids are the major species of cell membranes and removal of one of the FA results in the increase of lysophospholipids (LPL) usually through the enzymatic action of a phospholipase A2 (PLA2). Several studies reported the altered levels of phospholipase (PL) in neurodegenerative diseases concluding that secretary PLA2 activity in CSF might serve as a valuable biomarker of inflammation as demonstrated in Alzheimer's disease (Chalbot et al. 2009). In EAE model of SM, the blockage of PLA2 is highly efficacious in the amelioration of the disease courses probably by reducing $\mathrm{T}$ cell proliferation, pro-inflammatory cytokine production preventing activation of CNS microglia, and increasing myelin protein levels (Siritho and Freedman 2009).

\section{Conclusion}

The use of ${ }^{1} \mathrm{H}-\mathrm{NMR}$ spectroscopy was driven to obtain many compounds in CSF and to be able to carry out the identification of unknown compounds as well as to apply an easy robust methodology to be transferred into clinical practice. Analysis of metabolic profile of raw CSF and their lipid extract showed decreased levels of many compounds and led to the conclusion that MS patients could have a disturbance in FA synthesis as well as in other metabolic pathways perhaps leading to the decreased levels of acetyl-CoA. This, in turn, could reflect the disturbance processes of myelin regeneration and influence the neurotransmission processes (excitation/inhibition) due to energetic disturbances. Changes in the concentration of the compounds were detected using NMR-based metabolite profiles, and direction of those changes was in agreement with results of studies done by other research groups. Therefore, CSF metabolite profile analyses could be used as a fingerprint for early MS diagnosis.

Authors' Contribution A.P.P. contributed in MS patients recruit for study, patient group designed and describing, CSF samples collecting, participated in the draft of the manuscript.

A.K. contributed in control patients recruit for study, patient group designed and describing, CSF samples collecting, participated in the draft of the manuscript.

B.Z.P contributed in MS patients recruit for study, patient group designed and describing, CSF samples collecting.

M.N. contributed in MS patients recruit for study, patient group designed and describing, CSF samples collecting.

E.Z. contributed to the design of the study, prepared CSF samples for NMR experiments, contributed to interpreting the results, participated in the draft of the manuscript.

M.C. contributed to the design of the study, contributed to interpreting the results, participated in the draft of the manuscript.

B.T. contributed to the design of the study, measured and analyzed NMR spectra, performed the statistical analysis of all experimental data, contributed to interpreting the results and preparation of the figures, participated in the draft of the manuscript.

Authors A.P.P. and A.K. contributed equally to this work.

All authors revised and approved the final version of the manuscript.

Funding This research did not receive any specific grant from funding agencies in the public, commercial, or not-for-profit sectors.

\section{Compliance with Ethical Standards}

All procedures performed in studies involving human participants were in accordance with the ethical standards of the institutional researches committee and with the 1964 Helsinki declaration and its later amendments or compatible ethical standards.

Informed Consent Informed consent was obtained from all individual participants included in the study. 
Conflict of Interest The authors declare that they have no conflict of interest.

Open Access This article is distributed under the terms of the Creative Commons Attribution 4.0 International License (http:// creativecommons.org/licenses/by/4.0/), which permits unrestricted use, distribution, and reproduction in any medium, provided you give appropriate credit to the original author(s) and the source, provide a link to the Creative Commons license, and indicate if changes were made.

\section{References}

Balla T (2013) Phosphoinositides: tiny lipids with giant impact on cell regulation. Physiol Rev 93:1019-1137

Bendfeldt K, Kappos L, Radue EW, Borgwardt SJ (2009) Progression of gray matter atrophy and its association with white matter lesions in relapsing-remitting multiple sclerosis. J Neurol Sci 285:268-269 author reply 269

Bhargava P, Calabresi PA (2016) Metabolomics in multiple sclerosis. Mult Scler 22:451-460

Bo L, Dawson TM, Wesselingh S, Mork S, Choi S, Kong PA, Hanley D, Trapp BD (1994) Induction of nitric oxide synthase in demyelinating regions of multiple sclerosis brains. Ann Neurol 36:778-786

Brewer GJ, Wallimann TW (2000) Protective effect of the energy precursor creatine against toxicity of glutamate and beta-amyloid in rat hippocampal neurons. J Neurochem 74:1968-1978

Brosnan CF, Battistini L, Gao YL, Raine CS, Aquino DA (1996) Heat shock proteins and multiple sclerosis: a review. J Neuropathol Exp Neurol 55:389-402

Bylesjo M, Rantalainen M, Nicholson JK, Holmes E, Trygg J (2008) KOPLS package: kernel-based orthogonal projections to latent structures for prediction and interpretation in feature space. BMC Bioinformatics 9:106

Campbell GR, Mahad DJ (2012) Mitochondrial changes associated with demyelination: consequences for axonal integrity. Mitochondrion 12:173-179

Campbell GR, Ziabreva I, Reeve AK, Krishnan KJ, Reynolds R, Howell O, Lassmann H, Turnbull DM, Mahad DJ (2011) Mitochondrial DNA deletions and neurodegeneration in multiple sclerosis. Ann Neurol 69:481-492

Cha MH, Kim MJ, Jung J, Kim JH, Lee MS, Kim MS (2015) Metabolomic analysis of clinical plasma from cerebral infarction patients presenting with blood stasis. Evid Based Complement Alternat Med 2015:453423

Chalbot S, Zetterberg H, Blennow K, Fladby T, Grundke-Iqbal I, Iqbal K (2009) Cerebrospinal fluid secretory Ca2+-dependent phospholipase A2 activity is increased in Alzheimer disease. Clin Chem 55: 2171-2179

Cocco E, Murgia F, Lorefice L, Barberini L, Poddighe S, Frau J, Fenu G, Coghe G, Murru MR, Murru R, Del Carratore F, Atzori L, Marrosu MG (2016) (1)H-NMR analysis provides a metabolomic profile of patients with multiple sclerosis. Neurol Neuroimmunol Neuroinflamm 3:e185

Cornille E, Abou-Hamdan M, Khrestchatisky M, Nieoullon A, de Reggi M, Gharib B (2010) Enhancement of L-3-hydroxybutyryl-CoA dehydrogenase activity and circulating ketone body levels by pantethine. Relevance to dopaminergic injury. BMC Neurosci 11:51

DeGroot CJA, Ruuls SR, Theeuwes JWM, Dijkstra CD, VanderValk P (1997) Immunocytochemical characterization of the expression of inducible and constitutive isoforms of nitric oxide synthase in demyelinating multiple sclerosis lesions. J Neuropathol Exp Neurol $56: 10-20$
Del Boccio P, Rossi C, di Ioia M, Cicalini I, Sacchetta P, Pieragostino D (2016) Integration of metabolomics and proteomics in multiple sclerosis: from biomarkers discovery to personalized medicine. Proteomics Clin Appl 10:470-484

Dickens AM, Larkin JR, Davis BG, Griffin JL, Claridge TD, Sibson NR, Anthony DC (2015) NMR-based metabolomics separates the distinct stages of disease in a chronic relapsing model of multiple sclerosis. J NeuroImmune Pharmacol 10:435-444

Ellis DI, Dunn WB, Griffin JL, Allwood JW, Goodacre R (2007) Metabolic fingerprinting as a diagnostic tool. Pharmacogenomics 8:1243-1266

Fischer MT, Wimmer I, Hoftberger R, Gerlach S, Haider L, Zrzavy T, Hametner S, Mahad D, Binder CJ, Krumbholz M, Bauer J, Bradl M, Lassmann H (2013) Disease-specific molecular events in cortical multiple sclerosis lesions. Brain 136:1799-1815

Gonsette RE (2008) Neurodegeneration in multiple sclerosis: the role of oxidative stress and excitotoxicity. J Neurol Sci 274:48-53

Gonzalo H, Brieva L, Tatzber F, Jove M, Cacabelos D, Cassanye A, Lanau-Angulo L, Boada J, Serrano JC, Gonzalez C, Hernandez L, Peralta S, Pamplona R, Portero-Otin M (2012) Lipidome analysis in multiple sclerosis reveals protein lipoxidative damage as a potential pathogenic mechanism. J Neurochem 123:622-634

Hunter SF (2016) Overview and diagnosis of multiple sclerosis. Am J Manag Care 22:s141-s150

Joseph FG, Hirst CL, Pickersgill TP, Ben-Shlomo Y, Robertson NP, Scolding NJ (2009) CSF oligoclonal band status informs prognosis in multiple sclerosis: a case control study of 100 patients. J Neurol Neurosurg Psychiatry 80:292-296

Karp NA, Griffin JL, Lilley KS (2005) Application of partial least squares discriminant analysis to two-dimensional difference gel studies in expression proteomics. Proteomics 5:81-90

Keller JN, Mattson MP (1998) Roles of lipid peroxidation in modulation of cellular signaling pathways, cell dysfunction, and death in the nervous system. Rev Neurosci 9:105-116

Kim HH, Jeong IH, Hyun JS, Kong BS, Kim HJ, Park SJ (2017) Metabolomic profiling of CSF in multiple sclerosis and neuromyelitis optica spectrum disorder by nuclear magnetic resonance. PLoS One 12:e181758

Koneczny I, Cossins J, Vincent A (2014) The role of muscle-specific tyrosine kinase (MuSK) and mystery of MuSK myasthenia gravis. J Anat 224:29-35

Kuhle J, Disanto G, Dobson R, Adiutori R, Bianchi L, Topping J, Bestwick JP, Meier UC, Marta M, Dalla Costa G, Runia T, Evdoshenko E, Lazareva N, Thouvenot E, Iaffaldano P, Direnzo V, Khademi M, Piehl F, Comabella M, Sombekke M, Killestein J, Hegen H, Rauch S, D'Alfonso S, Alvarez-Cermeno JC, Kleinova P, Horakova D, Roesler R, Lauda F, Llufriu S, Avsar T, Uygunoglu U, Altintas A, Saip S, Menge T, Rajda C, Bergamaschi R, Moll N, Khalil M, Marignier R, Dujmovic I, Larsson H, Malmestrom C, Scarpini E, Fenoglio C, Wergeland S, Laroni A, Annibali V, Romano S, Martinez AD, Carra A, Salvetti M, Uccelli A, Torkildsen O, Myhr KM, Galimberti D, Rejdak K, Lycke J, Frederiksen JL, Drulovic J, Confavreux C, Brassat D, Enzinger C, Fuchs S, Bosca I, Pelletier J, Picard C, Colombo E, Franciotta D, Derfuss T, Lindberg R, Yaldizli O, Vecsei L, Kieseier BC, Hartung HP, Villoslada P, Siva A, Saiz A, Tumani H, Havrdova E, Villar LM, Leone M, Barizzone N, Deisenhammer F, Teunissen C, Montalban $\mathrm{X}$, Tintore M, Olsson T, Trojano M, Lehmann S, Castelnovo G, Lapin S, Hintzen R, Kappos L, Furlan R, Martinelli V, Comi G, Ramagopalan SV, Giovannoni G (2015) Conversion from clinically isolated syndrome to multiple sclerosis: a large multicentre study. Mult Scler 21:1013-1024

Lubina-Dabrowska N, Stepien A, Sulkowski G, Dabrowska-Bouta B, Langfort J, Chalimoniuk M (2017) Effects of IFN-betala and IFNbetalb treatment on the expression of cytokines, inducible NOS 
(NOS type II), and myelin proteins in animal model of multiple sclerosis. Arch Immunol Ther Exp 65:325-338

Lutz NW, Maillet S, Nicoli F, Viout P, Cozzone PJ (1998) Further assignment of resonances in $1 \mathrm{H}$ NMR spectra of cerebrospinal fluid (CSF). FEBS Lett 425:345-351

Moussallieh FM, Elbayed K, Chanson JB, Rudolf G, Piotto M, De Seze J, Namer IJ (2014) Serum analysis by $1 \mathrm{H}$ nuclear magnetic resonance spectroscopy: a new tool for distinguishing neuromyelitis optica from multiple sclerosis. Mult Scler 20:558-565

Navikas V, Link H (1996) Review: cytokines and the pathogenesis of multiple sclerosis. J Neurosci Res 45:322-333

Negre-Salvayre A, Auge N, Ayala V, Basaga H, Boada J, Brenke R, Chapple S, Cohen G, Feher J, Grune T, Lengyel G, Mann GE, Pamplona R, Poli G, Portero-Otin M, Riahi Y, Salvayre R, Sasson S, Serrano J, Shamni O, Siems W, Siow RC, Wiswedel I, Zarkovic K, Zarkovic N (2010) Pathological aspects of lipid peroxidation. Free Radic Res 44:1125-1171

Noga MJ, Dane A, Shi S, Attali A, van Aken H, Suidgeest E, Tuinstra T, Muilwijk B, Coulier L, Luider T, Reijmers TH, Vreeken RJ, Hankemeier T (2012) Metabolomics of cerebrospinal fluid reveals changes in the central nervous system metabolism in a rat model of multiple sclerosis. Metabolomics 8:253-263

O'Connor KC, Chitnis T, Griffin DE, Piyasirisilp S, Bar-Or A, Khoury S, Wucherpfennig KW, Hafler DA (2003) Myelin basic proteinreactive autoantibodies in the serum and cerebrospinal fluid of multiple sclerosis patients are characterized by low-affinity interactions. J Neuroimmunol 136:140-148

Pieragostino D, D'Alessandro M, di Ioia M, Rossi C, Zucchelli M, Urbani A, Di Ilio C, Lugaresi A, Sacchetta P, Del Boccio P (2015) An integrated metabolomics approach for the research of new cerebrospinal fluid biomarkers of multiple sclerosis. Mol BioSyst 11:15631572

Pieragostino D, Cicalini I, Lanuti P, Ercolino E, di Ioia M, Zucchelli M, Zappacosta R, Miscia S, Marchisio M, Sacchetta P, Onofrj M, Del Boccio P (2018) Enhanced release of acid sphingomyelinaseenriched exosomes generates a lipidomics signature in CSF of multiple sclerosis patients. Sci Rep 8:3071

Pietrocola F, Galluzzi L, Bravo-San Pedro JM, Madeo F, Kroemer G (2015) Acetyl coenzyme a: a central metabolite and second messenger. Cell Metab 21:805-821

Poddighe S, Murgia F, Lorefice L, Liggi S, Cocco E, Marrosu MG, Atzori L (2017) Metabolomic analysis identifies altered metabolic pathways in multiple sclerosis. Int J Biochem Cell Biol 93:148-155. https://doi.org/10.1016/j.biocel.2017.07.004

Poisson LM, Suhail H, Singh J, Datta I, Denic A, Labuzek K, Hoda MN, Shankar A, Kumar A, Cerghet M, Elias S, Mohney RP, Rodriguez M, Rattan R, Mangalam AK, Giri S (2015) Untargeted plasma metabolomics identifies endogenous metabolite with drug-like properties in chronic animal model of multiple sclerosis. J Biol Chem 290: 30697-30712

Polman CH, Reingold SC, Banwell B, Clanet M, Cohen JA, Filippi M, Fujihara K, Havrdova E, Hutchinson M, Kappos L, Lublin FD,
Montalban X, O'Connor P, Sandberg-Wollheim M, Thompson AJ, Waubant E, Weinshenker B, Wolinsky JS (2011) Diagnostic criteria for multiple sclerosis: 2010 revisions to the McDonald criteria. Ann Neurol 69:292-302

Raphael I, Webb J, Stuve O, Haskins W, Forsthuber T (2015) Body fluid biomarkers in multiple sclerosis: how far we have come and how they could affect the clinic now and in the future. Expert Rev Clin Immunol 11:69-91

Reinke SN, Broadhurst DL, Sykes BD, Baker GB, Catz I, Warren KG, Power C (2014) Metabolomic profiling in multiple sclerosis: insights into biomarkers and pathogenesis. Mult Scler 20:1396-1400

Ronowska A, Szutowicz A, Bielarczyk H, Gul-Hinc S, KlimaszewskaLata J, Dys A, Zysk M, Jankowska-Kulawy A (2018) The regulatory effects of acetyl-CoA distribution in the healthy and diseased brain. Front Cell Neurosci 12:169

Sharief MK (1991) Intrathecal synthesis of IgM in early multiple sclerosis. Acta Neurol Scand 84:456-457

Sinclair AJ, Viant MR, Ball AK, Burdon MA, Walker EA, Stewart PM, Rauz S, Young SP (2010) NMR-based metabolomic analysis of cerebrospinal fluid and serum in neurological diseases-a diagnostic tool? NMR Biomed 23:123-132

Siritho S, Freedman MS (2009) The prognostic significance of cerebrospinal fluid in multiple sclerosis. J Neurol Sci 279:21-25

Toczylowska B, Jamrozik Z, Liebert A, Kwiecinski H (2013) NMRbased Metabonomics of cerebrospinal fluid applied to amyotrophic lateral sclerosis. Bioprocess Biosyst Eng 33:21-32

Weiner HL (2008) A shift from adaptive to innate immunity: a potential mechanism of disease progression in multiple sclerosis. J Neurol 255(Suppl 1):3-11

Wevers RA, Engelke U, Wendel U, de Jong JG, Gabreels FJ, Heerschap A (1995) Standardized method for high-resolution 1H-NMR of cerebrospinal fluid. Clin Chem 41:744-751

Zhong S, Wang Y, Zhao G, Xiang Q, Ling X, Liu S, Huang L, Jia Y (2014) Similarities of biochemical abnormalities between major depressive disorder and bipolar depression: a proton magnetic resonance spectroscopy study. J Affect Disord 168:380-386

Zhou F, Zhuang Y, Gong H, Zhan J, Grossman M, Wang Z (2016) Resting state brain entropy alterations in relapsing remitting multiple sclerosis. PLoS One 11:e0146080

Zieminska E, Toczylowska B, Diamandakis D, Hilgier W, Filipkowski RK, Polowy R, Orzel J, Gorka M, Lazarewicz JW (2018) Glutamate, glutamine and GABA levels in rat brain measured using MRS, HPLC and NMR methods in study of two models of autism. Front Mol Neurosci 11:418

Publisher's Note Springer Nature remains neutral with regard to jurisdictional claims in published maps and institutional affiliations. 